\title{
Europaweite Umfrage Die meisten möchten bei schwerer Krankheit zu Hause sterben
}

9.344 Personen über 16 Jahre in England, Flandern, Deutschland, Italien, den Niederlanden, Portugal und Spanien wurden telefonisch gefragt, wo sie sterben wollten, wenn sie an einer schweren Krankheit mit weniger als einem Jahr Lebenserwartung litten. Sechs Möglichkeiten standen zur Auswahl: zu Hause, im Haus eines Angehörigen oder Freundes, im Hospiz oder einer palliativmedizinischen Einrichtung, in einer Klinik (ohne palliativmedizinische Betreuung), im Pflegeheim oder irgendwo anders.

Während es 64-84\% der Teilnehmer aller anderen Länder bevorzugten, zu Hause zu sterben, entschieden sich nur $50 \%$ der Portugiesen für einen Tod im eigenen Bett. Auch die Auswahlkriterien für den Sterbeort variierten je nach Nationalität. In Portugal, Spanien, den Niederlanden und Deutschland war der Wunsch daheim zu sterben altersabhängig. Beispielsweise wollten in Deutschland signifikant mehr Jüngere und über 70-Jährige zu Hause sterben als Befragte zwischen 60 und 69 Jahren. Engländer, Portugiesen, Spanier und Deutsche wollten ihren Sterbeplatz selbst wählen. Spanier und Deutsche hofften, so ihre positive Einstellung bewahren zu können. In Portugal und Flandern hatte Gewicht, dass anfallende Entscheidungen von der Familien mitgetragen werden könnten, wenn man selbst nicht mehr entscheidungsfähig ist. Die Autoren können nur spekulieren, warum so wenige Portugiesen zu Hause sterben wollten. Ein großer Respekt vor Autoritäten und ein starkes Sicherheitsbedürfnis könnten erklären, warum oft ein Tod in Klinik (8,3\%) oder Hospiz (35,7\%) bevorzugt wurde. Mit 83,1\% wollten die Niederländer - eventuell ein Spiegel der guten ambulanten Versorgungsmöglichkeiten schwer kranker Patienten in diesem Land - am häufigsten zu Hause sterben. In Deutschland gaben 66\% der Befragten an, bei einer schweren Erkrankung zu Hause sterben zu wollten. 24,8\% der Probanden bevorzugten ein Hospiz oder eine palliative Einrichtung und nur 3,4\% wählten ein Krankenhaus.

\section{Christine Starostzik}

Gomes B et al. Preferences for place of death if faced with advanced cancer: a population survey in England, Flanders, Germany, Italy, the Netherlands, Portugal and Spain. Ann Oncol. 2012 Feb 16. [Epub ahead of print].

\section{Schilddrüsenkrebserkrankungen nehmen zu Anstieg durch iodiertes Salz bedingt?}

Die jährliche Inzidenz von Schilddrüsenkarzinomen stieg in Dänemark von 1943-2008 von 0,41 auf 1,57 pro 100.000 Männer, von 0,90 auf 4,11 pro 100.000 Frauen und damit die Zahl der Neuerkrankungen im Durchschnitt um 1,7 bzw. 1,8 \% jährlich. Am stärksten war der Zuwachs in den Jahren nach 1992, so lag etwa zwischen 2001 und 2008 die jährliche Steigerung bei 6,0 bzw. 4,0\%. Dabei ließ sich die Zunahme der Schilddrüsenkarzinominzidenz fast ausschließlich auf die Zunahme von papillären Karzinomen zurückführen, denn follikuläre und medulläre Karzinome blieben stabil, anaplastische Tumoren gingen zurück. Neben der Verschiebung der Tumortypen zeigte sich, dass die Patienten jünger wurden. Spiegeln diese Registerzahlen einen realen Zuwachs oder nur die Fortschritte in der Diagnostik von Schilddrüsenkarzinomen wider?

Die genauen Ursachen konnten die Autoren nicht identifizieren, halten es aber für denkbar, dass die seit 2000 in Dänemark vorgeschriebene Iodierung von Haushaltssalz zu der erhöhten Krebsrate beitrug. Auch in anderen Ländern seien nach der Einführung von Iodsupplementierungs-Programmen papilläre Schilddrüsenkarzinome häufiger beobachtet worden. Daten aus Schottland, wo die Inzidenz des Schilddrüsenkrebses auch ohne Iodsupplementierung zugenommen hat, schwächen die Hypothese. Gegen eine gesteigerte Iodzufuhr als einzige Ursache spricht auch der Zeitpunkt: Die Zahl der Krebserkrankungen ging bereits vor dem Jahr 2000 nach oben. Eine Belastung mit radioaktiver Strahlung aus Nuklearanlagen schließen die Studienautoren für Dänemark als Ursache aus. Zu einem erhöhten Risiko für Schilddrüsenkrebs könnten aber Chemikalien wie die beispielsweise in Plastik enthaltenen polybromierten Diphenylether (PBDE) oder ein Selenmangel sowie die immer häufiger schon bei Kindern angefertigten Röntgenaufnahmen von Kiefer und Zähnen beitragen. Nicht auszuschließen ist, dass durch die intensivere Diagnostik klinisch irrelevante, nicht tastbare Tumoren häufiger festgestellt werden.

\section{Beate Schumacher}

Blomberg M et al. Thyroid cancer in Denmark 1943-2008, before and after iodine supplementation. Int J Cancer. 2012 Feb 15. [Epub ahead of print]

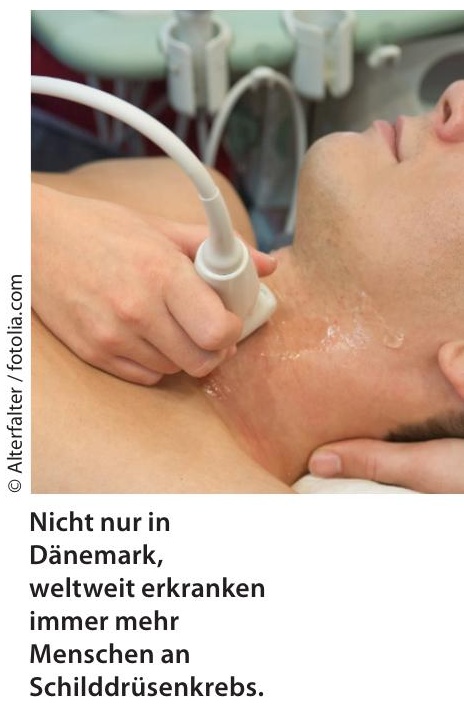

Schilddrüsenkrebs. 